\title{
Microhomology Selection for Microhomology Mediated End Joining in Saccharomyces cerevisiae
}

\author{
Kihoon Lee ${ }^{1}$, Jae-Hoon $\mathrm{Ji}^{2}$, Kihoon Yoon ${ }^{3}$, Jun Che ${ }^{3}$, Ja-Hwan Seol ${ }^{1}$, Sang Eun Lee ${ }^{1,3, *(\mathbb{D})}$ and \\ Eun Yong Shim ${ }^{3, *}$ \\ 1 Department of Molecular Medicine, Institute of Biotechnology, \\ University of Texas Health Science Center at San Antonio, 7703 Floyd Curl Drive, \\ San Antonio, TX 78229-3900, USA; y2k0108@gmail.com (K.L.); seolj@uthscsa.edu (J.-H.S.) \\ 2 Genomic Instability Research Center, Ajou University School of Medicine, 164, World Cup-ro, \\ Yeongtong-gu, Suwon 16499, Korea; jij@ajou.ac.kr \\ 3 Department of Radiation Oncology, University of Texas Health Science Center at San Antonio, \\ 7703 Floyd Curl Drive, San Antonio, TX 78229-3900, USA; yoon.kihoon@hotmail.com (K.Y.); \\ chej@uthscsa.edu (J.C.) \\ * Correspondence: lees4@uthscsa.edu (S.E.L.); shim@uthscsa.edu (E.Y.S.); \\ Tel.: +1-210-562-4157 (S.E.L.); +1-210-562-4162 (E.Y.S.); Fax: +1-210-562-4161 (S.E.L. \& E.Y.S.)
}

Received: 4 March 2019; Accepted: 28 March 2019; Published: 8 April 2019 updates

\begin{abstract}
Microhomology-mediated end joining (MMEJ) anneals short, imperfect microhomologies flanking DNA breaks, producing repair products with deletions in a $\mathrm{Ku}$ - and RAD52-independent fashion. Puzzlingly, MMEJ preferentially selects certain microhomologies over others, even when multiple microhomologies are available. To define rules and parameters for microhomology selection, we altered the length, the position, and the level of mismatches to the microhomologies flanking homothallic switching $(\mathrm{HO})$ endonuclease-induced breaks and assessed their effect on MMEJ frequency and the types of repair product formation. We found that microhomology of eight to 20 base pairs carrying no more than 20\% mismatches efficiently induced MMEJ. Deletion of MSH6 did not impact MMEJ frequency. MMEJ preferentially chose a microhomology pair that was more proximal from the break. Interestingly, MMEJ events preferentially retained the centromere proximal side of the $\mathrm{HO}$ break, while the sequences proximal to the telomere were frequently deleted. The asymmetry in the deletional profile among MMEJ products was reduced when $\mathrm{HO}$ was induced on the circular chromosome. The results provide insight into how cells search and select microhomologies for MMEJ in budding yeast.
\end{abstract}

Keywords: microhomology; microhomology-mediated end joining; DNA double strand break; mismatch; deletion

\section{Introduction}

DNA double strand breaks (DSBs) are a lethal lesion type that can lead to catastrophic cellular outcomes if not repaired quickly and accurately. Multiple mechanisms were evolved to remove DNA DSBs. Two of the most well-known DSB repair mechanisms are homologous recombination (HR) and classical non-homologous end joining (C-NHEJ), which repair DNA breaks by copying from an undamaged template across the lesion or re-ligation of broken ends after juxtaposition of broken ends together, respectively [1,2]. The third, poorly defined DSB repair mechanisms, called microhomology-mediated end joining (MMEJ) or alternative end joining (A-EJ), remove DNA breaks via annealing of microhomology flanking DSB, yielding repair products with intervening sequence deletion between microhomology pairs [3-6]. 
Because of the high mutation burden and chromosome structure changes that accompany MMEJ, MMEJ should be tightly regulated in order to serve as an alternative repair option when C-NHEJ or HR are deficient. Nevertheless, MMEJ or A-EJ operate universally in eukaryotic cells and induce a range of DNA aberrations, including telomere fusions and chromosomal rearrangements [7-10]. In yeast, DNA DSBs with no complementary overhangs produce repair events with junctional microhomology (5-15 bps) and the size of deletion ranging from a few to kilobases of nucleotides [11,12]. Oligonucleotide-mediated joining of non-complementary DNA ends and circularization of extra-chromosomal URA4 fragments in fission yeast are also mediated by microhomology flanking DNA ends and are Ku and Lig4-independent [13]. In mammals, repair junctions containing microhomology (albeit shorter than those found in yeast) were found in $\mathrm{Ku}$ or Lig4 mutants during the repair of enzyme-induced break or B-and T-cell receptor gene rearrangements [14,15]. The repair of P-element excised breaks in flies and telomere fusions in plants also occurs in Lig4-independent with the repair products featuring substantial microhomologies at the junctions [16-18]. Evidence also suggests that the repair of Cas9 or Talen induced DNA DSBs could be mediated by flanking microhomology with the deletion of intervening sequences [19].

Biochemically, MMEJ depends on a set of proteins, many of which participate in other repair pathways, such as base excision repair or HR $[3,5,6,20]$. In yeast, the mechanistic similarity between NHEJ, single strand annealing (SSA), and MMEJ helps reveal a few potential biochemical steps in MMEJ [12]. According to this model, DSB is first processed to generate $3^{\prime}$ single stranded DNA by Mre11 and Sae2-dependent nuclease activity and to reveal embedded microhomology flanking DSB. Microhomology is then brought together by an unidentified manner independent of RAD52 or Rad59 [10,12]. Heterotrimeric Replication Protein A (RPA) complex inhibits microhomology annealing and MMEJ [21,22]. Any non-homologous tails formed during annealing of microhomology are then cleaved by Rad1/Rad10 endonuclease, and the remaining gap is filled in by a collection of enzymes including Pol3 and Pol4 [11,12]. The nick is then ligated by Dnl4 and Cdc9-dependent manner [11]. In mammals, MMEJ depends on Pol $\theta$ and LigIII for repair synthesis and ligation, respectively $[8,23]$. MMEJ also depends on PARP1 and XRCC3 [24].

MMEJ is an emerging drug target due to its role as the back-up DSB repair pathway in C-NHEJor HR-deficient cancers [25-28]. Pol $\theta$ is highly expressed in ovarian cancer cells, and inactivation of Pol $\theta$ is synthetic to $B R C A 2$ deficiency. Recently, inactivation of FEN1 caused BRCA2 synthetic growth defect due likely to its role in MMEJ [29]. Systematic and comprehensive analyses of the biochemical and the genetic components of MMEJ in multiple models are thus warranted and would facilitate the development of rationalized treatment strategies for wide ranges of cancers. Deciphering the rules and parameters of microhomology engagement and annealing should help decipher MMEJ mechanisms and allow us to predict the types of repair junctions in events associated with DNA damage and assessment of precise contributions of MMEJ to chromosomal instability in many biological events.

One of the key steps in MMEJ is microhomology annealing; yet, how cells select certain microhomology over others is unknown to date. MMEJ in yeast occurs at a lower frequency than in mammalian cells and requires higher stringency for stable pairing between paired microhomologies. The basis for this stringent microhomology annealing in budding yeast is unknown to date.

In this study, we systematically analyzed the features of microhomology in model MMEJ reporters by altering the position, the length, and the degree of homology in microhomology. We then measured MMEJ frequency and types of MMEJ products using genetic approaches. The results revealed insights into how cells select certain microhomology for MMEJ and key parameters in dictating the types of MMEJ products in yeast.

\section{Material and Methods}

\subsection{Yeast Strains and Plasmids}

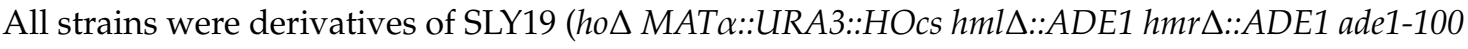
leu2-3,112 lys5 trp1::hisG ura3-52 ade3::GAL::HO)(Table 1) [30]. To construct the MMEJ reporter with 
different lengths and sequences of microhomology pairs, the endogenous $M A T$ locus was replaced with MAT::URA3::HO cut site fragment of pSL19 [12] with the desired modifications introduced by different oligonucleotide pairs for PCR amplifications. The gene deletion mutants were generated by the PCR-derived KANMX module. The yeast strain (R072) with a circular chromosome III was a gift from Dr. James Haber [31], and the MAT locus was replaced with the MAT::HO::URA3 fragment from pSL19 using the one step gene replacement technique.

Table 1. Strain List.

\begin{tabular}{|c|c|}
\hline SLY19 & 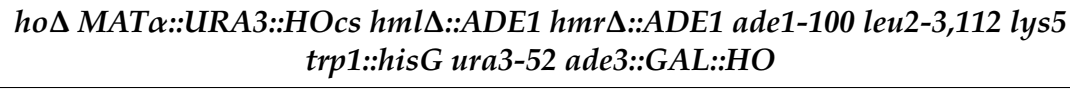 \\
\hline YKHL76 & SLY19 ku70 $:: K A N$ \\
\hline YKHL77 & SLY19 RAD1 $\triangle:: K A N$ \\
\hline YKHL101 & SLY19 12bp microhomology $(M H) \Delta$ \\
\hline YKHL102 & JKM179 MAT::URA3::HO::6MH \\
\hline YKHL103 & YKHL102 ku70 $::$ KAN \\
\hline YKHL104 & YKHL102 RAD1 $:: K A N$ \\
\hline YKHL105 & JKM179 MAT::URA3::HO::10MH \\
\hline YKHL106 & YKHL105 ku70ه::KAN \\
\hline YKHL107 & YKHL105 RAD1 $:: K A N$ \\
\hline YKHL108 & JKM179 MAT::URA3::HO::12MH \\
\hline YKHL109 & YKHL108 ku70A::KAN \\
\hline YKHL111 & YKHL108 RAD1 $:: K A N$ \\
\hline YKHL112 & JKM179 MAT::URA3::HO::15MH \\
\hline YKHL113 & YKHL112 ku70 $:: K A N$ \\
\hline YKHL114 & YKHL112 RAD1 $\triangle:: K A N$ \\
\hline YKHL115 & JKM179 MAT::URA3::HO::18MH \\
\hline YKHL116 & YKHL115 ku70 $::$ KAN \\
\hline YKHL117 & YKHL115 RAD1 $\triangle:: K A N$ \\
\hline YKHL118 & JKM179 MAT::URA3::HO::20MH \\
\hline YKHL119 & YKHL118 ku70 $::$ KAN \\
\hline YKHL121 & YKHL118 RAD1 $:: K A N$ \\
\hline YKHL122 & YKHL118 rad52 $\Delta:: K A N$ \\
\hline YKHL123 & JKM179 MAT::URA3::HO::25MH \\
\hline YKHL124 & YKHL123 ku70 $:: K A N$ \\
\hline YKHL125 & YKHL123 $\mathrm{r} a d 1 \Delta:: K A N$ \\
\hline YKHL126 & YKHL123 $\mathrm{rad52} \Delta:: K A N$ \\
\hline YKHL127 & JKM179 MAT::URA3::HO::29MH \\
\hline YKHL128 & YKHL127 ku70 $\Delta:: K A N$ \\
\hline YKHL129 & YKHL127 RAD1 $:: K A N$ \\
\hline YKHL131 & YKHL127 rad52 \\
\hline YKHL132 & JKM179 MAT::URA3::HO::200bp::12MH \\
\hline YKHL133 & YKHL132 ku70 $:: K A N$ \\
\hline YKHL134 & YKHL132 RAD1 $\triangle:: K A N$ \\
\hline YKHL142 & JKM179 MAT::URA3::HO::12MH::200bp::12MH \\
\hline YKHL143 & YKHL142 ku70A::KAN \\
\hline YKHL144 & YKHL142 RAD1 $:: K A N$ \\
\hline YKHL145 & JKM179 MAT::URA3::HO::12MH::20bp::12MH \\
\hline YKHL146 & YKHL145 ku70 $:: K A N$ \\
\hline YKHL147 & YKHL145 RAD1 $\triangle:: K A N$ \\
\hline YKHL148 & JKM179 MAT::URA3::HO::12MH w/ mismatch gggtttataGGattatactg \\
\hline YKHL149 & YKHL148 ku70 $:: K A N$ \\
\hline YKHL151 & YKHL148 RAD1 $\triangle:: K A N$ \\
\hline YJHJ1 & YKHL148 msh6 $\Delta:: K A N$ \\
\hline YKHL152 & JKM179 MAT::URA3::HO::12MH w/ mismatch gggtttataaaattGGactg \\
\hline YKHL153 & YKHL152 ku70 ::KAN \\
\hline YKHL154 & YKHL152 RAD1 $\triangle:$ KAN \\
\hline YJHJ2 & YKHL152 msh6 \\
\hline
\end{tabular}


Table 1. Cont.

\begin{tabular}{|c|c|}
\hline SLY19 & 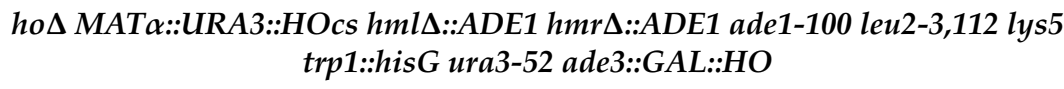 \\
\hline YKHL155 & JKM179 MAT::URA3::HO::12MH w/ mismatch ggCAttataaaattatactg \\
\hline YKHL156 & YKHL155 ku70A::KAN \\
\hline YKHL157 & YKHL155 RAD1 $\triangle:: K A N$ \\
\hline YJHJ3 & YKHL155 msh6 \\
\hline YKHL158 & JKM179 MAT::URA3::HO::12MH w/ mismatch ggCtttataTaattaAactg \\
\hline YKHL159 & YKHL158 ku70 $:: K A N$ \\
\hline YKHL161 & YKHL158 RAD1 $:: K A N$ \\
\hline YJHJ4 & YKHL158 msh6 $\Delta:: K A N$ \\
\hline YKHL165 & JKM179 MAT::URA3::HO::12MH w/mismatch gAgttAatTaaaGtatCctg \\
\hline YKHL166 & YKHL165 ku70 \\
\hline YKHL167 & YKHL165 RAD1 $:: K A N$ \\
\hline YKHL179 & YKHL152 msh6 $\triangle:: K A N$ \\
\hline YKHL182 & JKM179 MAT::URA3::12MH-1::12MH-2::HO::12MH-1::12MH-2 \\
\hline YKHL183 & YKHL182 ku70A::KAN \\
\hline YKHL184 & YKHL182 RAD1 $:: K A N$ \\
\hline YKHL185 & JKM179 MAT::URA3::12MH::12MH::HO::12MH \\
\hline YKHL186 & YKHL185 ku70 $\Delta:: K A N$ \\
\hline YKHL187 & YKHL185 RAD1 $:: K A N$ \\
\hline YKHL188 & JKM179 MAT::URA3::17MH::12MH::HO::17MH::12MH \\
\hline YKHL189 & YKHL188 ku70 $::$ KAN \\
\hline YKHL201 & YKHL188 RAD1 $:: K A N$ \\
\hline YKHL202 & JKM179 MAT::URA3::17MH::12MH::HO::17MH::12MH \\
\hline YKHL203 & YKHL188 ku70 $::$ KAN \\
\hline YKHL204 & YKHL188 RAD1 $\triangle:: K A N$ \\
\hline YKHL208 & JKM179 MATA Mup1::12MH-2::12MH-1::HO::12MH-2::12MH-1 \\
\hline YKHL209 & JKM179 MATA Mup1::12MH-1::12MH-2::HO::12MH-1::12MH-2 \\
\hline R072 & described at Haber and Thorburn (1984) \\
\hline YKHL211 & R072 circular ChIII MAT::URA3::HO::12MH::20bp::12MH \\
\hline
\end{tabular}

\subsection{Homothallic Switching Endonuclease Induction}

Cells were grown in pre-induction YEP-glycerol media for overnight at $30^{\circ} \mathrm{C}$, and serial dilutions of cells grown to mid-log phase were plated onto YEP-agar medium containing either galactose (YEP-GAL), which induces galactose inducible homothallic switching endonuclease (HO) expression to generate DSBs, or glucose (YEPD). Survival frequency was calculated by dividing the number of colonies growing on YEP-GAL by the number of colonies growing on YEPD.

\subsection{Analysis of Repair Events}

Colonies growing on YEP-GAL plates were replica plated onto synthetic complete (SC) medium lacking uracil, and the mating type was determined by complementation test using tester strains. Uracil auxotrophs that were a mating type were selected for analyzing the repair events by amplifying the region spanning the repair junctions with PCR using a set of primers- $\mathrm{pX}$ (5'-GTAAACGGTGTCCTCTGTAAGG-3') and p2 (5'-TCGAAAGATAAACAACCTCC-3') - and then subjected to sequencing [11]. To examine deletional bias of DNA ends in MMEJ products, PCR of the repair products was performed using primer $\mathrm{P} 2$ and $\mathrm{pI}$ (5'-CACTCTACAAAACCAAAACCAGGG).

\section{Results}

\subsection{Microhomology is not Randomly Selected for MMEJ}

Enzymatic cleavage at two inversely oriented $\mathrm{HO}$ cut sites separated by $\sim 2.0 \mathrm{~kb} U R A 3$ at the MAT locus of yeast chromosome III generated four base $3^{\prime}$ DNA overhangs with no complementary end sequences and whose repair relied heavily $(>80 \%)$ on $\mathrm{Ku}$ - and $R A D 52$-independent as well as 
Rad1-dependent MMEJ (SLY19, Figure 1A,B) [11,12]. Less than 20\% of the repair still depended on Ku-dependent imprecise NHEJ (Figure 1B). Analysis of repair products revealed that the majority of the repair events (34/46 sequenced, see Table 1 in Ma et al., 2003) involved annealing of a microhomology pair composed of 12-nucleotide sequences with two mismatch bases, each situated at 2-bp centromere proximal and 60-bp distal from the breaks. Puzzlingly, in silico analysis for the available microhomologies at sequences flanking DNA break spanning several kilobases of sequences identified over three hundred microhomology pairs that were energetically as favorable as the one used predominantly in our assay (Figure S1). Furthermore, no essential genes were present at the regions spanning $7 \mathrm{~kb}$ and $0.5 \mathrm{~kb}$ from either side of the break to prevent the usage of other microhomology pairs for MMEJ. Therefore, the rarity of MMEJ events involving other microhomology pairs could not simply be explained by the lack of other microhomology at sequences flanking an HO induced DSB.
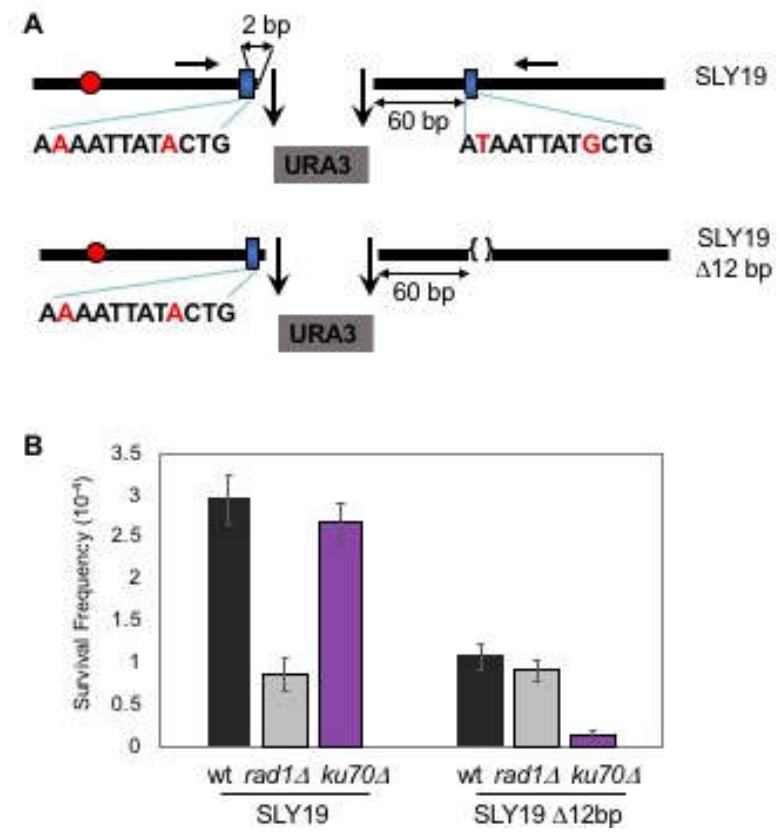

Figure 1. Microhomology selection for microhomology-mediated end joining (MMEJ) is non-random. (A) Two homothallic switching endonuclease (HO) cleavages at the MAT locus (the 117-bp MATa and the full-length $M A T \alpha$ cut sites) separated by $\sim 2 \mathrm{~kb}$ of $U R A 3$ sequence preferentially induced $\mathrm{Ku}$ and RAD52-independent Microhomology-mediated end joining (MMEJ) using the flanking 12-bp of imperfect microhomology that was 2-bp and 60-bp away from the break. The location of the centromere (red circles) and the preferred microhomology (blue boxes) for MMEJ are shown. The sequence (mismatches are shown in red characters) of the preferred microhomology are shown below the blue boxes. The positions of the primers for PCR amplification and sequencing are shown by red arrows. (B) Survival frequency after induction of HO breaks of the wild type (WT), the RAD1 gene deleted [RAD1 $\Delta$ representing the frequency of classical non-homologous end joining (C-NHEJ)], and the KU70 deletion derivatives ( $k u 70 \Delta$, the frequency of MMEJ) of SLY19 or SLY19 lacking preferred 12-bp imperfect microhomology (SLY19 $\Delta 12-b p)$. The frequency of survival after an HO-induced double strand breaks (DSB) was calculated by dividing the number of colonies growing on YEP-agar medium containing galactose (YEP-GAL) by the number of colonies growing on YEP-agar medium containing glucose (YEPD). Each value represents the average from at least three independent experiments \pm standard deviation.

To further define how a cell selects certain microhomology for MMEJ, we deleted 12-bp microhomology proximal to the telomeric side in SLY19 and forced cells to choose other available microhomology pairs for MMEJ (Figure 1B). Surprisingly, the deletion of 12-bp microhomology, despite it being energetically less stable than others, almost completely eliminated MMEJ events (the events 
occurring in Ku70 deficient cells), leaving the remaining repair dependent on Ku-dependent NHEJ only (the events in RAD1 deleted cells) (Figure 1B). Other microhomologies identified based on their energy levels and the sizes (over 12-bp) by in silico analysis failed to substitute deleted microhomology for MMEJ. The results reinforce the view that cells favor certain microhomology over others for MMEJ and underscore the importance of defining the rules and the parameters for microhomology selection.

\subsection{MMEJ Requires Microhomology of 8- to 20-Nucleotide Long}

As the first step toward defining the microhomology selection rules in MMEJ, we determined the optimum size of microhomology for MMEJ by substituting the 12-nucleotide imperfect microhomology to the 6-25 nucleotide of perfect microhomology in SLY19 and measured the frequency of MMEJ after induction of an HO DSB. The types of repair events from the survivors were analyzed by PCR amplification and sequencing of the region spanning the repair junctions using a set of oligonucleotides that annealed the $5^{\prime}$ to the 117-bp MATa cleavage site and the $3^{\prime}$ to the MAT $\alpha$ HO recognition site (Figure 1A). Strains deleted for $Y K U 70, R A D 1$, or RAD52 gene were analyzed in parallel to deduce the type of repair mechanisms.

We discovered that microhomology shorter than 8-bp did not produce MMEJ at a detectable level, and the repair occurred almost exclusively by Ku-dependent imprecise NHEJ only (Figure 2). In contrast, the repair of DNA break flanked by microhomology longer than 20-bp became partially dependent on RAD52 and corresponded to SSA. Between 8-20 nucleotide-microhomology, MMEJ frequency increased linearly as the size of microhomology increased. Ku-dependent C-NHEJ events remained constant in all strains regardless of the sizes of microhomology (Figure 2). We concluded that an efficient MMEJ requires 8- to 20-bp microhomology in yeast. We previously reached a similar conclusion from the MMEJ reporter with complementary $3^{\prime}$ ends [10], indicating that the size preference is universal to MMEJ in yeast.
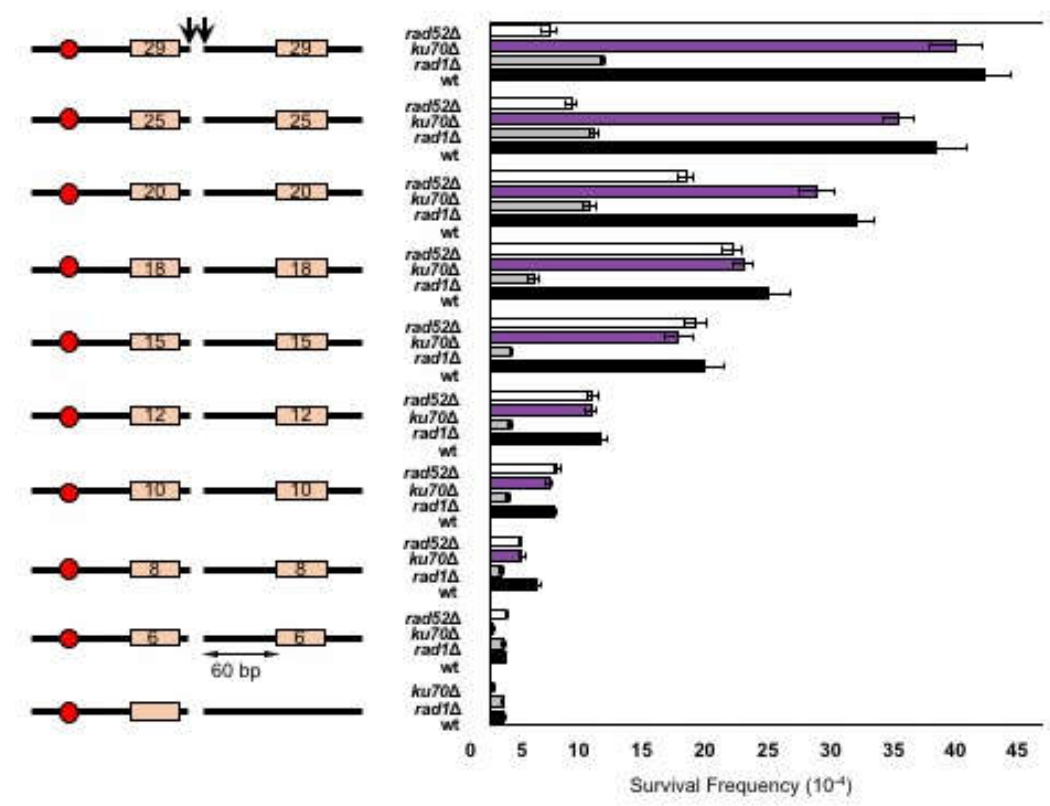

Figure 2. MMEJ requires 8-20 bp microhomology. (Left) A diagram of MMEJ reporters with different sizes of microhomology flanking an $\mathrm{HO}$ break. The locations of HO cut site (arrow), microhomology (orange boxes), and centromere (red circle) are shown. The size of microhomology is shown in number (bp) inside the microhomology. (Right) Graph showing survival frequency \pm standard deviation (SD) in wild type (WT), RAD1 $\triangle$ (MMEJ deficient mutant), ku70 (C-NHEJ deficient mutant), and RAD52 $\Delta$ [homologous recombination (HR) deficient mutant]. Survival frequency was calculated by dividing the number of colonies surviving on the YEP-GAL plates by the number of colonies surviving on the YEPD plates. The results are the average of three independent experiments. 


\subsection{Effect of Distance between Microhomology and DNA Break on Microhomology Usage}

Next, we tested if the proximity from the break dictates the frequency of microhomology selection and annealing in MMEJ. To test this, we deleted the 12-bp microhomology sequence at 60-bp distal from the break in SLY19 and relocated it to either 260-bp or 1.4-kb away from the break (Figure 3). We then measured the MMEJ frequency after $\mathrm{HO}$ expression in different repair pathway gene mutants. We found that relocating the 12-bp microhomology to 1.4-kb away from the break reduced MMEJ frequency $(11.4 \%)$ compared to that when microhomology was located at an original location $(78.2 \%)$ or at 260 -bp distal to the break $(67.5 \%)$, suggesting that the distance from the break represents a key parameter in dictating the frequency of MMEJ.
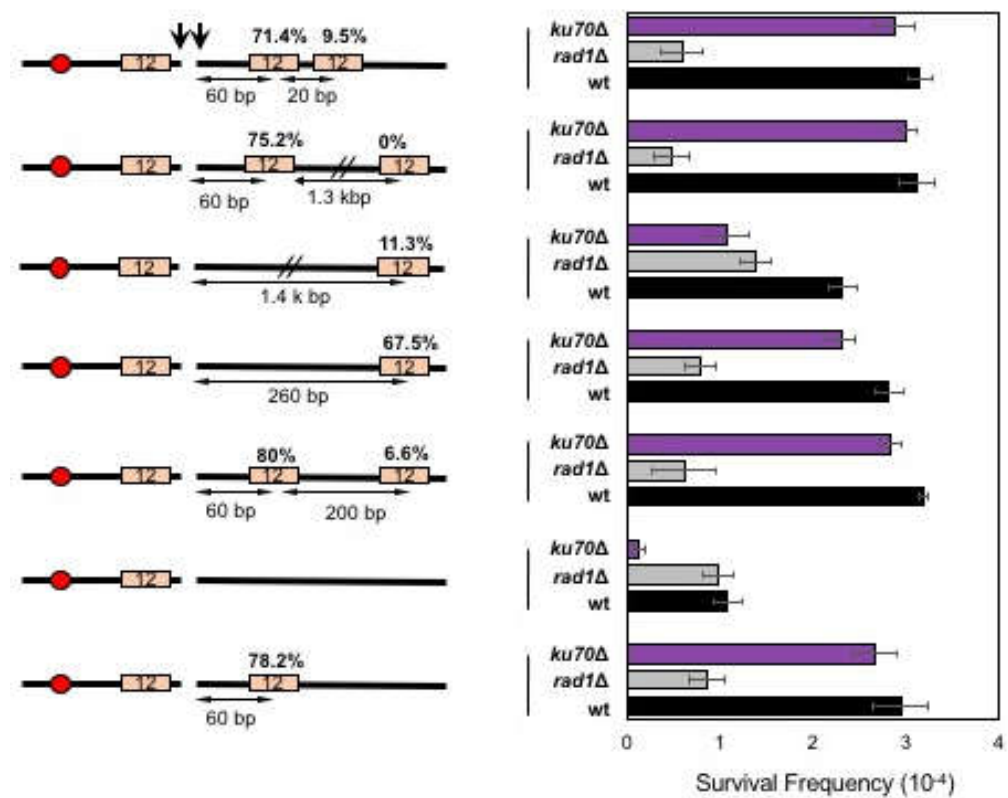

Figure 3. The effect of proximity on microhomology selection for MMEJ. (Left) A diagram of MMEJ reporters with microhomologies located at different distances to an $\mathrm{HO}$ break. The locations of $\mathrm{HO}$ cut site (arrow), microhomology (orange boxes), and centromere (red circles) are shown. The size of microhomology is shown in number (bp) inside the microhomology. The distance to the break (bp) is shown below each microhomology. Percentage of repair event was calculated by dividing the number of repair events using each microhomology with the number of all repair events, regardless of the repair types and shown above each microhomology. The repair events were analyzed by sequencing of the repair junctions from $>100$ Ura- survivors. (Right) Graph showing survival frequency \pm SD in wild type (WT), RAD1 $\triangle$ (MMEJ deficient mutant), and ku70 $\Delta$ (C-NHEJ deficient mutant). Survival frequency was calculated as described in Figure 1. The results are the average of three independent experiments.

To further analyze the effect of microhomology position on microhomology selection and MMEJ frequency, we generated yeast strains carrying two identical microhomologies that were telomere proximal to the break; one located at 60-bp (the original location) and the other at 260- or 1400-bp distal from the break site (Figure 3). We then examined if the distally located microhomology could successfully compete to more proximally located microhomology for MMEJ. When cells were presented with two competing microhomologies for MMEJ, proximal microhomology was used more frequently $(75.2 \%$ or $80 \%)$, leaving the distal microhomology usage to only $0-6.6 \%$ among all repair events (Figure 3).

The preferential use of proximal microhomology could be explained by the end resection that exposed the proximal microhomology prior to the distally located one [2]. To test this idea, we placed two identical microhomologies that were only 20-bp apart at the telomere proximal side of the break and assessed their usage for MMEJ after $\mathrm{HO}$ expression. According to the current estimation of resection 
rate $(4 \mathrm{~kb} / \mathrm{h})$ [32], there should have been less than a 20 second difference in resecting proximal and distal microhomologies. We predicted that if the end resection dictated the proximity effect of microhomology selection in MMEJ, the longer the distance between competing microhomologies would lead to more bias in microhomology selection for MMEJ. However, even when two microhomologies were only 20-bp apart, cells still retained the proximity bias (71.4\%) similar to those between microhomologies $200-\mathrm{bp}$ or $1.3-\mathrm{kb}$ apart. The results suggest that cells preferentially use proximal microhomology for MMEJ repair.

\subsection{Effect of Mismatches on Microhomology Selection}

The 12-bp microhomology used overwhelmingly in our assay contained two mismatch bases (Figure 1A). We tested if the presence of mismatches within microhomology influenced the microhomology selection in MMEJ or dictated the types of repair events. To test this question, we replaced the telomere proximal 12-bp microhomology to the 20-bp microhomology with 1-, 2-, 3-, and 5-bp mismatches at various positions in SLY19 (Figure 4A). We then measured repair frequency upon induction of $\mathrm{HO}$ breaks. The 20-bp microhomology helped detect more broad ranges of MMEJ deficiencies. The longer microhomology also allowed additional room for variations in the position and the number of mismatches within the preferred microhomology in our assay.
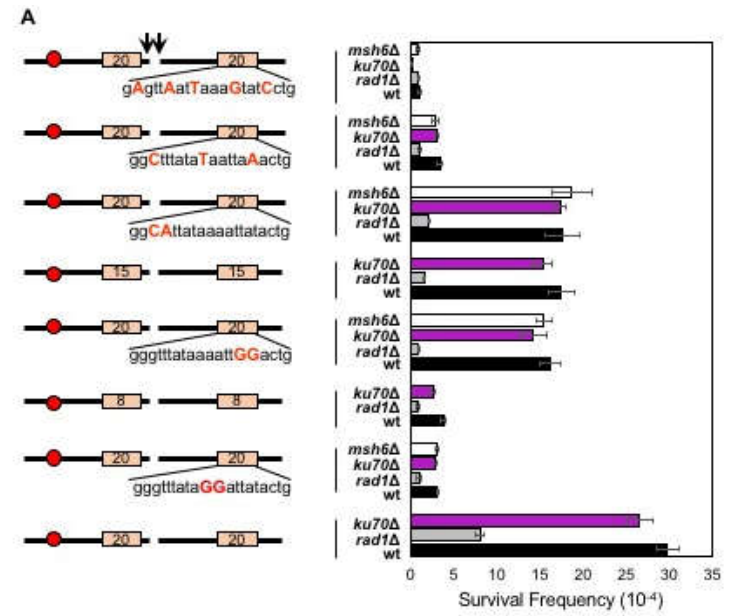

B

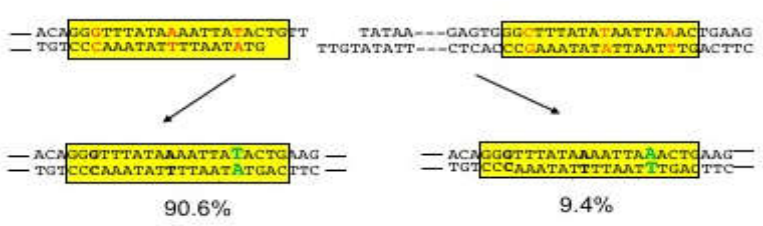

Figure 4. The effect of mismatches on microhomology selection for MMEJ. (A) Left: A diagram of MMEJ reporters with imperfect microhomologies carrying different numbers and positions of mismatches. The locations of HO cut site (arrow), microhomology (orange boxes), and centromere (red circles) are shown. The size of microhomology is shown in number (bp) inside the microhomology. The sequence of microhomology and the position of mismatch (highlighted in red) are shown. Right: Graph showing survival frequency \pm s.d. in wild type (WT), RAD1 $\triangle$ (MMEJ deficient mutant), ku70 $\Delta$ (C-NHEJ deficient mutant), and $m s h 6 \Delta$ (mismatch repair deficient mutant). Survival frequency was calculated as described in Figure 1. The results are the average of three independent experiments. (B) Two different ways (highlighted in green characters) mismatches (shown in red) in microhomology were repaired. The corrected bases are shown in bold characters. The microhomology is shown in yellow boxes. Analyses of $94 \mathrm{MMEJ}$ products showed strong bias to one type over the other. The percentage of repair product type among total MMEJ repair events was calculated based on sequencing of the repair junctions from the Ura- survivors after $\mathrm{HO}$ expression. 
The incorporation of one to three mismatched nucleotides gradually decreased MMEJ frequency such that three base mismatches significantly (almost five-fold) reduced MMEJ frequency (Figure 4A). The five mismatches completely disabled MMEJ, leaving all repair events Ku-dependent imprecise NHEJ. Interestingly, the positions of mismatches also affected the MMEJ frequency; when two consecutive mismatches were present, the mismatches essentially reduced the size of microhomology, and the frequency of MMEJ was identical to that of the longer remaining microhomology between the two (for instance, two GG mismatches at positions 15 and 16 rendered cells to repair HO break at a frequency similar to that of 15 -bp microhomology).

To test if the inhibitory effect of mismatches on MMEJ was due to the action of the mismatch repair mechanism, yeast cells deleted for MSH6 were constructed, and MMEJ frequency with two to five mismatched microhomology was determined. Msh2-Msh6 catalyzed heteroduplex rejection to limit homeologous recombination and correct base pair mismatches [33]. We found that deletion of MSH6 did not alter MMEJ frequency when cells were provided with the mismatched microhomology pairs (Figure 4A). The results suggest that mismatch repair does not play detectable roles in microhomology selection and MMEJ repair.

\subsection{Biased Mismatch Correction in Imperfect Microhomology-Mediated MMEJ}

The mismatches within microhomology could generate two different types of MMEJ products bearing junctional sequence identical to telomere proximal or distal microhomology (Figure 4B). We analyzed the MMEJ products using two primers flanking microhomology pair with three mismatches and subjected them to sequence analysis. Surprisingly, distinct bias existed on the types of MMEJ products-among MMEJ events with three nucleotide mismatches, the final MMEJ products preferentially $(90.6 \%)$ kept the full nucleotide sequence of the centromeric proximal microhomology, and even the remaining $10 \%$ carried two of the three mismatches identical to centromere proximal microhomology (Figure 4B).

\subsection{MMEJ Produces Repair Products Preferentially Deleting the Telomere Proximal Side of the Break}

The correction bias to retain centromere proximal side of the microhomology prompted us to test if the position of microhomology on either side of the break (centromeric or telomeric side) affected microhomology usage or the types of MMEJ products. To test this idea, yeast strains (SLY19 derivatives) were constructed to carry 12- or 17- bp microhomology pairs that were located at the same distance from the break but caused the deletion of different sides of the break by MMEJ (Figure 5). We then measured the MMEJ frequency and type of the repair products after $\mathrm{HO}$ induction and sequence analysis.

Surprisingly, cells almost exclusively used microhomology pairs to minimize the deletion at the centromeric side of the break for MMEJ (Figure 5). When yeast strains were induced to MMEJ using 12- or 17-bp microhomology that deleted 60-bp to the centromeric side of the break, MMEJ was dramatically reduced. Even when we placed two (12- and 17-bp) microhomology pairs flanking the break with the longer microhomology (17-bp) positioned to lead centromeric side deletion, cells rarely (6.6\%) used 17-bp microhomology in MMEJ (Figure 5). The results suggest that cellular selection of microhomology pair for MMEJ depends on the location of microhomology. 

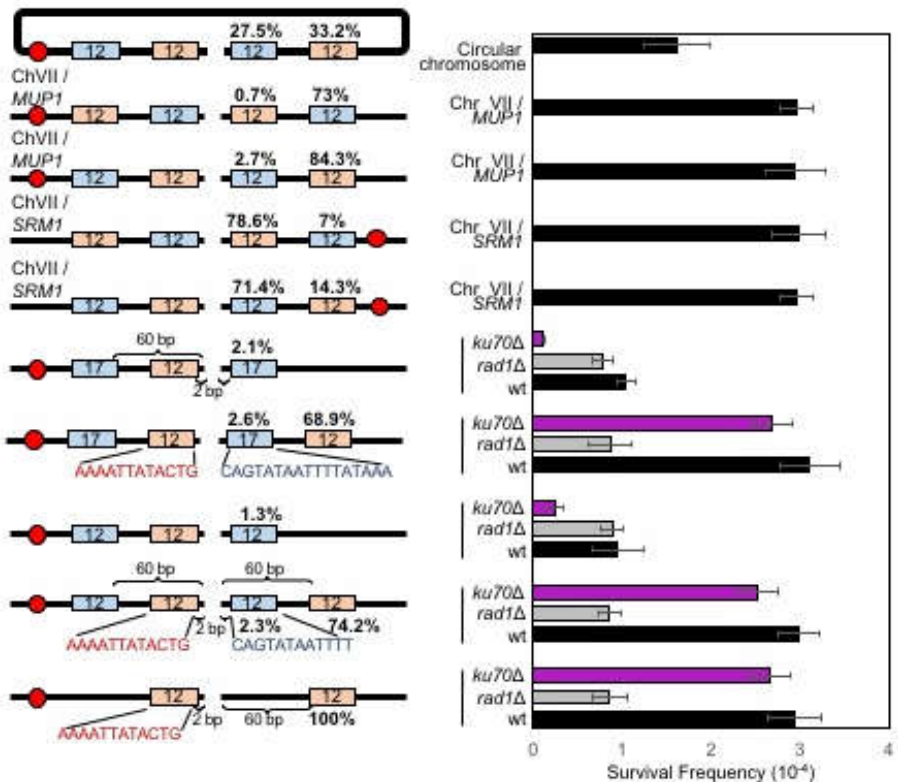

Figure 5. The position effect of microhomology on MMEJ. Left: A diagram of MMEJ reporters with microhomology pairs at different locations to the HO break. The locations of $\mathrm{HO}$ cut site (arrow), microhomology (orange and blue boxes), and centromere (red circles) are shown. The size of microhomology is shown in number (bp) inside the microhomology. The distance to the break (bp) is shown above each microhomology. The size of the $3^{\prime}$ flap (bp) and the sequences of microhomologies are also shown. Percentage of repair event was calculated by dividing the number of repair events using each microhomology with the number of all repair events, regardless of the repair types and shown above each microhomology. The repair events were analyzed by sequencing of the repair junctions from >100 Ura- survivors. Right: Graph showing survival frequency \pm s.d. in wild type (WT), RAD1 $\Delta$ (MMEJ deficient mutant), and $k u 70 \Delta$ (C-NHEJ deficient mutant). Survival frequency was calculated as described in Figure 1. The results are the average of three independent experiments.

\subsection{Chromosome Circularization Disrupts Microhomology Selection Bias in MMEJ}

We considered if the observed bias in MMEJ product formation reflected unique chromatin landscapes of the chromosome III. To test this possibility, we relocated our MMEJ reporter with two competing 12-bp microhomology pairs at each arm of chromosome VII (next to SRM1 or MUP1) in two different orientations (Figure 5). We found that the bias in MMEJ product type persisted even at new locations, and MMEJ associated deletion was preferential to the telomeric side of the DSB end (Figure 5). The results suggest that the asymmetric deletion and the biased MMEJ product type formation is not unique to chromosome III.

We then surmised if the selection of microhomology for MMEJ was influenced by the location of the nearby telomere sequences. To test this idea, we constructed yeast strain carrying our MMEJ reporter with two microhomology pairs at the MAT locus of a circular chromosome III. Circular chromosome III was generated by the fusion of $H M L$ and $H M R$ and completely lacked a telomeric sequence (Figure 5) [31]. We then measured MMEJ frequency and analyzed the type of repair products by sequencing. Surprisingly, we no longer detected microhomology selection bias (44.7 versus $55.3 \%$ ) from MMEJ events in the circular chromosome, even though the overall MMEJ frequency was reduced to $75 \%$ of the linear chromosome (Figure 5).

\section{Discussion}

Microhomology annealing is central to MMEJ events and dictates the frequency and types of MMEJ products. MMEJ mysteriously uses certain microhomology sequences over others, but we do not know how cells choose microhomologies or what dictates this decision process. The lack of 
such information prevents us from making accurate predictions of the repair junction types in events associated with DNA damage and assessment of precise contributions of MMEJ to chromosomal instability in many biological events. In this report, we partially filled this gap of knowledge using the MMEJ reporter that almost exclusively uses 12-bp imperfect microhomology for MMEJ [12]. Our results show that the distance and the size of the microhomologies are two key parameters of microhomology selection in MMEJ.

In our experiments, MMEJ preferentially used proximal 8- to 20-bp microhomology. The repair events with longer microhomology (> $20 \mathrm{bp}$ ) became partially RAD52-dependent and thus could be mediated by SSA. We previously reported a similar size requirement on microhomology for MMEJ using a different MMEJ reporter bearing a single DSB [10]. The precise reason for $>20$ bp homology requirement of SSA is unknown; it could be related to the heptameric structure of $R A D 52$, as each subunit binds ssDNA that is four nucleotides long [34,35]. RAD52 might not be able to bind to microhomology less than 20-bp for SSA. Similarly, we described the effect of distance between microhomology and DNA break on MMEJ before [10], but used the assay system without competing microhomology pairs. Our study thus provides the first evidence that cells preferentially use closer microhomology for MMEJ, even when two competing microhomologies are only 20-bp apart. The apparent preference on proximal microhomology for MMEJ can be explained by their ordered availability in annealing, as the end resection proceeds linearly from the break to the distal side. However, in this scenario, two microhomologies that are 20-bp apart should be resected almost simultaneously, and the distance effect should be less apparent than when microhomologies are 200-bp or 1.3-kb apart. Alternatively, the proximity preference of microhomology selection can be attributed to the size of the $3^{\prime}$ flap, as the MMEJ events using distally located microhomology entail longer $3^{\prime}$ flaps. A great deal of evidence indicates that the size of $3^{\prime}$ flaps is the key determinant for efficient break-induced replication and inter-chromosomal MMEJ [36,37]. We also demonstrated that deletion of RAD1/RAD10, the key $3^{\prime}$ flap nuclease, abrogates MMEJ in several MMEJ reporters [10-12]. We can envision that long $3^{\prime}$ flaps might destabilize annealing of short microhomology. Indeed, Pol32 is specifically required for MMEJ involving longer $3^{\prime}$ flaps and shorter microhomology [10].

We previously showed that MMEJ frequency follows first order enzyme kinetics and depends on the melting temperature of available microhomologies [38]. Accordingly, any mismatches in microhomologies reduce the melting temperature of microhomology and MMEJ frequency [10,38]. Overall, MMEJ frequency corresponds well to the melting temperature of the given microhomologies, even though the location of mismatches also affects MMEJ frequency. The effect of mismatch positions on MMEJ is unknown to date. We discovered that Msh6 does not impact on the frequency of MMEJ using imperfect microhomologies. Interestingly, mismatches in microhomology are corrected with puzzling bias to one type over the other [37]. Although we do not know the molecular basis of this bias, the same process might control the retention of the centromeric side of the break over the telomeric side and dictate the microhomology selection and the repair types.

How does a cell preferentially retain one end during MMEJ? Evidence suggests that repair proteins are asymmetrically recruited to two DNA ends during DSB repair [39]. Association of Rad51 initiates close to the break but shows asymmetric binding pattern flanking the DSB [39]. H2A.Z distribution is also asymmetrical at the centromeric and the telomeric side of the break [39]. Notably, we reported that deletion of RAD52 did not affect the MMEJ frequency but altered the types of MMEJ products [11]. It should be possible that asymmetric association of RAD52 or other repair factors at DSB might modulate microhomology selection/annealing and the types of repair products. We also propose that repair protein distribution is altered in circular chromosome to attenuate the deletional bias during MMEJ. Additional experiments are necessary to identify factors responsible for end retention bias in MMEJ and their distributions in linear and circular chromosomes. 


\section{Conclusions}

Taken together, we propose that selection of microhomology for MMEJ is non-random and likely follows certain rules and parameters to maximize repair efficiency and fidelity. The size, the position, and the mismatches of microhomology in aggregate dictate the repair outcome in conjunction with a not yet identified mechanism to retain one end of the break for MMEJ. Elucidation of the underlying mechanism of directional and positional bias in microhomology selection is likely important and would shed light on MMEJ-mediated chromosomal aberrations and mutagenesis.

Supplementary Materials: The following are available online at http://www.mdpi.com/2073-4425/10/4/284/s1, Figure S1: Distribution of 12-mer microhomology pairs flanking HO cleavage site.

Author Contributions: K.L., J.-H.J., K.Y., S.E.L. and E.Y.S. designed the experiments. K.L., J.-H.J., J.C., and J.-H.S. constructed the yeast strains and performed the genetic assays. K.L., J.-H.J., K.Y., J.C., J.-H.S., S.E.L. and E.Y.S. analyzed the data and wrote the paper.

Funding: This research was funded by William and Ella Owens Medical Research Foundation, Nathan Shock Center Pilot grant, and NIH research grant GM71011 to S.E.L., and ThriveWell Foundation to E.Y.S.

Acknowledgments: We are grateful to Jim Haber for providing yeast strain with circular chromosome III.

Conflicts of Interest: The authors declare no conflict of interest.

\section{References}

1. San Filippo, J.; Sung, P.; Klein, H. Mechanism of eukaryotic homologous recombination. Annu. Rev. Biochem. 2008, 77, 229-257. [CrossRef] [PubMed]

2. Symington, L.S.; Gautier, J. Double-strand break end resection and repair pathway choice. Annu. Rev. Genet. 2011, 45, 247-271. [CrossRef]

3. Seol, J.H.; Shim, E.Y.; Lee, S.E. Microhomology-mediated end joining: Good, bad and ugly. Mutat. Res. 2017, 809, 81-87. [CrossRef] [PubMed]

4. Sfeir, A.; Symington, L.S. Microhomology-Mediated End Joining: A Back-up Survival Mechanism or Dedicated Pathway? Trends Biochem. Sci. 2015, 40, 701-714. [CrossRef]

5. Sinha, S.; Villarreal, D.; Shim, E.Y.; Lee, S.E. Risky business: Microhomology-mediated end joining. Mutat. Res. 2016, 788, 17-24. [CrossRef] [PubMed]

6. Wang, H.; Xu, X. Microhomology-mediated end joining: New players join the team. Cell Biosci. 2017, 7, 6 . [CrossRef]

7. Lee-Theilen, M.; Matthews, A.J.; Kelly, D.; Zheng, S.; Chaudhuri, J. CtIP promotes microhomology-mediated alternative end joining during class-switch recombination. Nat. Struct. Mol. Biol. 2011, 18, 75-79. [CrossRef] [PubMed]

8. Mateos-Gomez, P.A.; Gong, F.; Nair, N.; Miller, K.M.; Lazzerini-Denchi, E.; Sfeir, A. Mammalian polymerase theta promotes alternative NHEJ and suppresses recombination. Nature 2015, 518, 254-257. [CrossRef]

9. Simsek, D.; Brunet, E.; Wong, S.Y.; Katyal, S.; Gao, Y.; McKinnon, P.J.; Lou, J.; Zhang, L.; Li, J.; Rebar, E.J.; et al. DNA ligase III promotes alternative nonhomologous end-joining during chromosomal translocation formation. PLoS Genet. 2011, 7, e1002080. [CrossRef]

10. Villarreal, D.D.; Lee, K.; Deem, A.; Shim, E.Y.; Malkova, A.; Lee, S.E. Microhomology directs diverse DNA break repair pathways and chromosomal translocations. PLoS Genet. 2012, 8, e1003026. [CrossRef]

11. Ma, J.L.; Kim, E.M.; Haber, J.E.; Lee, S.E. Yeast Mre11 and Rad1 proteins define a Ku-independent mechanism to repair double-strand breaks lacking overlapping end sequences. Mol. Cell. Biol. 2003, 23, 8820-8828. [CrossRef]

12. Lee, K.; Lee, S.E. Saccharomyces cerevisiae Sae2- and Tel1-dependent single-strand DNA formation at DNA break promotes microhomology-mediated end joining. Genetics 2007, 176, 2003-2014. [CrossRef] [PubMed]

13. Decottignies, A. Alternative end-joining mechanisms: A historical perspective. Front. Genet. 2013, 4, 48. [CrossRef]

14. Liang, L.; Deng, L.; Nguyen, S.C.; Zhao, X.; Maulion, C.D.; Shao, C.; Tischfield, J.A. Human DNA ligases I and III, but not ligase IV, are required for microhomology-mediated end joining of DNA double-strand breaks. Nucleic Acids Res. 2008, 36, 3297-3310. [CrossRef] [PubMed] 
15. Yan, C.T.; Boboila, C.; Souza, E.K.; Franco, S.; Hickernell, T.R.; Murphy, M.; Gumaste, S.; Geyer, M.; Zarrin, A.A.; Manis, J.P.; et al. IgH class switching and translocations use a robust non-classical end-joining pathway. Nature 2007, 449, 478-482. [CrossRef] [PubMed]

16. Heacock, M.L.; Idol, R.A.; Friesner, J.D.; Britt, A.B.; Shippen, D.E. Telomere dynamics and fusion of critically shortened telomeres in plants lacking DNA ligase IV. Nucleic Acids Res. 2007, 35, 6490-6500. [CrossRef]

17. McVey, M.; Lee, S.E. MMEJ repair of double-strand breaks (director's cut): Deleted sequences and alternative endings. Trends Genet. 2008, 24, 529-538. [CrossRef] [PubMed]

18. Yu, A.M.; McVey, M. Synthesis-dependent microhomology-mediated end joining accounts for multiple types of repair junctions. Nucleic Acids Res. 2010, 38, 5706-5717. [CrossRef] [PubMed]

19. Sakuma, T.; Nakade, S.; Sakane, Y.; Suzuki, K.T.; Yamamoto, T. MMEJ-assisted gene knock-in using TALENs and CRISPR-Cas9 with the PITCh systems. Nat. Protoc. 2016, 11, 118-133. [CrossRef]

20. Howard, S.M.; Yanez, D.A.; Stark, J.M. DNA damage response factors from diverse pathways, including DNA crosslink repair, mediate alternative end joining. PLoS Genet. 2015, 11, e1004943. [CrossRef]

21. Deng, S.K.; Gibb, B.; de Almeida, M.J.; Greene, E.C.; Symington, L.S. RPA antagonizes microhomology-mediated repair of DNA double-strand breaks. Nat. Struct. Mol. Biol. 2014, 21, 405-412. [CrossRef]

22. McVey, M. RPA puts the brakes on MMEJ. Nat. Struct. Mol. Biol. 2014, 21, 348-349. [CrossRef] [PubMed]

23. Kent, T.; Chandramouly, G.; McDevitt, S.M.; Ozdemir, A.Y.; Pomerantz, R.T. Mechanism of microhomology-mediated end-joining promoted by human DNA polymerase theta. Nat. Struct. Mol. Biol. 2015, 22, 230-237. [CrossRef]

24. Soni, A.; Siemann, M.; Grabos, M.; Murmann, T.; Pantelias, G.E.; Iliakis, G. Requirement for Parp-1 and DNA ligases 1 or 3 but not of Xrcc1 in chromosomal translocation formation by backup end joining. Nucleic Acids Res. 2014, 42, 6380-6392. [CrossRef] [PubMed]

25. Ceccaldi, R.; Liu, J.C.; Amunugama, R.; Hajdu, I.; Primack, B.; Petalcorin, M.I.; O'Connor, K.W.; Konstantinopoulos, P.A.; Elledge, S.J.; Boulton, S.J.; et al. Homologous-recombination-deficient tumours are dependent on Poltheta-mediated repair. Nature 2015, 518, 258-262. [CrossRef] [PubMed]

26. Ledermann, J.A. PARP inhibitors in ovarian cancer. Ann. Oncol. 2016, 27 (Suppl. 1), i40-i44. [CrossRef]

27. Ledermann, J.A.; Drew, Y.; Kristeleit, R.S. Homologous recombination deficiency and ovarian cancer. Eur. J. Cancer 2016, 60, 49-58. [CrossRef] [PubMed]

28. Liu, F.W.; Tewari, K.S. New Targeted Agents in Gynecologic Cancers: Synthetic Lethality, Homologous Recombination Deficiency, and PARP Inhibitors. Curr. Treat. Opt. Oncol. 2016, 17, 12. [CrossRef]

29. Mengwasser, K.E.; Adeyemi, R.O.; Leng, Y.; Choi, M.Y.; Clairmont, C.; D'Andrea, A.D.; Elledge, S.J. Genetic Screens Reveal FEN1 and APEX2 as BRCA2 Synthetic Lethal Targets. Mol. Cell 2019, 73, 885.e6-899.e6. [CrossRef] [PubMed]

30. Lee, S.E.; Moore, J.K.; Holmes, A.; Umezu, K.; Kolodner, R.D.; Haber, J.E. Saccharomyces Ku70, mre11/rad50 and RPA proteins regulate adaptation to G2/M arrest after DNA damage. Cell 1998, 94, 399-409. [CrossRef]

31. Haber, J.E.; Thorburn, P.C. Healing of broken linear dicentric chromosomes in yeast. Genetics 1984, 106, 207-226.

32. Zhu, Z.; Chung, W.H.; Shim, E.Y.; Lee, S.E.; Ira, G. Sgs1 helicase and two nucleases Dna2 and Exo1 resect DNA double-strand break ends. Cell 2008, 134, 981-994. [CrossRef] [PubMed]

33. Sugawara, N.; Goldfarb, T.; Studamire, B.; Alani, E.; Haber, J.E. Heteroduplex rejection during single-strand annealing requires Sgs1 helicase and mismatch repair proteins Msh2 and Msh6 but not Pms1. Proc. Natl. Acad. Sci. USA 2004, 101, 9315-9320. [CrossRef] [PubMed]

34. Parsons, C.A.; Baumann, P.; Van Dyck, E.; West, S.C. Precise binding of single-stranded DNA termini by human RAD52 protein. EMBO J. 2000, 19, 4175-4181. [CrossRef]

35. Stasiak, A.Z.; Larquet, E.; Stasiak, A.; Muller, S.; Engel, A.; Van Dyck, E.; West, S.C.; Egelman, E.H. The human Rad52 protein exists as a heptameric ring. Curr. Biol. 2000, 10, 337-340. [CrossRef]

36. Anand, R.; Beach, A.; Li, K.; Haber, J. Rad51-mediated double-strand break repair and mismatch correction of divergent substrates. Nature 2017, 544, 377-380. [CrossRef] [PubMed]

37. Meyer, D.; Fu, B.X.; Heyer, W.D. DNA polymerases delta and lambda cooperate in repairing double-strand breaks by microhomology-mediated end-joining in Saccharomyces cerevisiae. Proc. Natl. Acad. Sci. USA 2015, 112, E6907-E6916. [CrossRef] 
38. Sinha, S.; Li, F.; Villarreal, D.; Shim, J.H.; Yoon, S.; Myung, K.; Shim, E.Y.; Lee, S.E. Microhomology-mediated end joining induces hypermutagenesis at breakpoint junctions. PLoS Genet. 2017, 13, e1006714. [CrossRef]

39. Kalocsay, M.; Hiller, N.J.; Jentsch, S. Chromosome-wide Rad51 spreading and SUMO-H2A.Z-dependent chromosome fixation in response to a persistent DNA double-strand break. Mol. Cell 2009, 33, 335-343. [CrossRef]

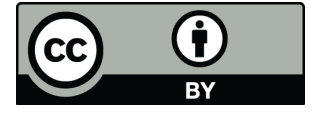

(C) 2019 by the authors. Licensee MDPI, Basel, Switzerland. This article is an open access article distributed under the terms and conditions of the Creative Commons Attribution (CC BY) license (http://creativecommons.org/licenses/by/4.0/). 